International Journal of Algebra, Vol. 8, 2014, no. 3, 135 - 147

HIKARI Ltd, www.m-hikari.com

http://dx.doi.org/10.12988/ija.2014.416

\title{
Some New Inequalities on Geometric Multiplicities and Geršgorin Discs
}

\author{
Rachid Marsli \\ Department of Mathematics and Statistics \\ Georgia State University \\ Atlanta, GA 30303, USA \\ Frank J. Hall \\ Department of Mathematics and Statistics \\ Georgia State University \\ Atlanta, GA 30303, USA
}

Copyright (c) 2014 Rachid Marsli and Frank J. Hall. This is an open access article distributed under the Creative Commons Attribution License, which permits unrestricted use, distribution, and reproduction in any medium, provided the original work is properly cited.

\begin{abstract}
In this paper the authors continue their work on geometric multiplicities and Geršgorin discs done in a series of four recent papers. The new results involve principal submatrices, an upper bound on the absolute value of an eigenvalue, the rank of a matrix, non-real eigenvalues, and powers of matrices. Some consequences of the results and examples are provided.
\end{abstract}

mathematics Subject Classification: $15 \mathrm{~A} 18$

Keywords: geometric multiplicity; Geršgorin disc

\section{Introduction}

We denote the set of all $n \times n$ complex matrices by $M_{n}$. In this paper all matrices are complex unless otherwise qualified. One of the most attractive 
and useful results to locate the eigenvalues of a matrix is Geršgorin's theorem, which goes back to 1931. The main part of this theorem is the following.

Geršgorin Theorem. Let $A \in M_{n}$ and let

$$
R_{i}^{\prime}=\sum_{j=1, j \neq i}^{n}\left|a_{i j}\right|, \quad 1 \leq i \leq n
$$

denote the deleted absolute row sums of $A$. Then every eigenvalue of $A$ is located in the union of its $n$ Geršgorin discs (G-discs)

$$
\cup_{i=1}^{n} D_{i}
$$

where

$$
D_{i}=\left\{z \in C:\left|z-a_{i i}\right| \leq R_{i}^{\prime}\right\}
$$

A matrix $A \in M_{n}$ has $n$ Geršgorin discs $D_{i}$, some of which may degenerate into points and some of which may be duplicates, as in the trivial example of an identity matrix. Recently, the authors extended the Geršgorin theory in the articles [1], [3], [4], and [5]. In particular, the following result was proved in $[3]$.

Theorem 1.1. Let $\lambda$ be an eigenvalue of the matrix $A \in M_{n}$ with geometric multiplicity $k$. Then $\lambda$ is in at least $k$ of the Geršgorin discs $D_{i}$ of $A$.

More recently, we obtained a refinement of Theorem 1.1 in [5].

Theorem 1.2. Let $\lambda$ be an eigenvalue of the matrix $A \in M_{n}$ with geometric multiplicity at least $k \geq 1$. Construct the $n \times n$ matrix $C_{k}$ in the following way: in every row of $A$, replace the smallest $k-1$ off-diagonal entries in absolute value by zeros. Then $\lambda$ is in at least $k$ of the Geř̆gorin discs of $C_{k}$.

In particular, two corollaries were mentioned in [5].

Corollary 1.3. Let $\lambda$ be an eigenvalue of the matrix $A \in M_{n}$ with geometric multiplicity at least $k \geq 1$. Then

$$
\lambda \in \cup_{j=1}^{n-k+1}\left\{z \in C:\left|z-a_{i_{j} i_{j}}\right| \leq R_{i_{j}}^{\prime}\left(C_{k}\right)\right\}
$$

for any choice of indices $1 \leq i_{1}<\ldots<i_{n-k+1} \leq n$. There are $\left(\begin{array}{c}n \\ k-1\end{array}\right)$ possibilities for such a union, so that $\lambda$ is contained in their intersection.

The next one is an immediate corollary of Theorem 1.2, based again on the $\operatorname{matrix} C_{k}$. 
Corollary 1.4. Let $A \in M_{n}$ and let $k \geq 1$. If each collection of $G$-discs of the matrix $C_{k}$ that is separated from the remaining $G$-discs of $C_{k}$ consists of at most $k-1$ discs, then each eigenvalue of $A$ has geometric multiplicity less than $k$. In particular, if this is the case for $k=2$, then each eigenvalue of $A$ has geometric multiplicity 1, that is, $A$ is nonderogatory.

In the following sections, we denote matrices $C_{i}$ constructed from the $n \times n$ matrix $A$ in the same way as in Theorem 1.2: in every row of $A$, replace the smallest $i-1$ off-diagonal entries in absolute value by zeros.

In this paper we continue our work on geometric multiplicities and Geršgorin discs. The new results involve principal submatrices, an upper bound on the absolute value of an eigenvalue, the rank of a matrix, non-real eigenvalues, and powers of matrices. In fact, we build upon several results from our earlier articles. Some consequences of the new results and examples are provided.

\section{Geometric multiplicity and principal sub- matrices}

In Theorem 1.4.10 in [2] the authors prove the following result.

Theorem 2.1. Let $\lambda$ be an eigenvalue of the $n \times n$ matrix $A$ with geometric multiplicity at least $k$. If $\hat{A}$ is an $m \times m$ principal submatrix of $A$ and if $m>n-k$, then $\lambda$ is an eigenvalue of $\hat{A}$.

We can give a lower bound on the geometric multiplicity of $\lambda$ as an eigenvalue of $\hat{A}$.

Theorem 2.2. Let $\lambda$ be an eigenvalue of the matrix $A \in M_{n}$ with geometric multiplicity at least $k$ and let $\hat{A} \in M_{m}$ be a principal submatrix of $A$ with $m>n-k$. Then the geometric multiplicity of $\lambda$ as an eigenvalue of $\hat{A}$ is at least $m+k-n$.

Proof. It suffices to prove the result for an eigenvalue $\lambda=0$; for $\lambda \neq 0$ one can then use the matrix $A-\lambda I$. So, take $\lambda=0$. Observe that since

$$
\text { nullity } A \geq k \text {, }
$$

we have

$$
\operatorname{rank} A \leq n-k,
$$

so that

$$
\operatorname{rank} \hat{A} \leq n-k \text {. }
$$

Thus,

$$
\text { nullity } \hat{A} \geq m-n+k \text {. }
$$


Corollary 2.3. Let $A \in M_{n}$, let $\hat{A} \in M_{m}$ be a principal submatrix of $A$, let $1 \leq l \leq m$, and suppose that $\hat{A}$ has no eigenvalue with geometric multiplicity strictly larger than $l$. Then no eigenvalue of $A$ has geometric multiplicity strictly larger than $n+l-m$.

Proof. If $\lambda$ is an eigenvalue of $A$ with geometric multiplicity $k>n+l-m$, then $k+m>n+l-m+m=n+l>n$. By Theorem 2.2, $\lambda$ is an eigenvalue of $\hat{A}$ with geometric multiplicity $\geq k+m-n>n+l-m+m-n=l$. This is a contradiction.

With the use of both Theorems 1.2 and 2.2, the following result has a similar proof.

Corollary 2.4. Let $A \in M_{n}$ with $n \geq 2$, let $\hat{A} \in M_{m}$ be a principal submatrix of $A$ with $m \geq 2$, and suppose that $C_{2}(\hat{A})$ has disjoint Geršgorin discs. Then no eigenvalue of $A$ has geometric multiplicity strictly larger than $n+1-m$.

Example 2.5. Consider the matrices

$\hat{A}=\left[\begin{array}{rrrr}-2 & 1 / 4 & 1 / 2 & 1 / 2 \\ 1 / 4 & 1 & -1 & 1 / 2 \\ -1 / 2 & -1 / 2 & 4 & 1 / 2 \\ 1 / 2 & -1 / 2 & 1 & 7\end{array}\right]$ and $A=\left[\begin{array}{rrrrrr}-2 & 1 / 4 & 1 / 2 & 1 / 2 & * & * \\ 1 / 4 & -1 & 1 / 2 & * & * \\ -1 / 2 & -1 / 2 & 4 & 1 / 2 & * & * \\ 1 / 2 & -1 / 2 & 1 & 7 & * & * \\ * & * & * & * & * & * \\ * & * & * & * & * & *\end{array}\right]$.

$C_{2}(\hat{A})$ has disjoint Geršgorin discs; hence it cannot have any eigenvalue with geometric multiplicity strictly larger than 1. Thus, by Corollary 2.4, A cannot have any eigenvalue with geometric multiplicity strictly larger than $3=$ $6+1-4$.

Remark 2.6. Corollary 2.4 provides an algorithm that may determine an upper bound for the geometric multiplicities of the eigenvalues of a matrix A. If this algorithm encounters any principal submatrix $\hat{A}$ of order $m$ such that $C_{2}(\hat{A})$ has disjoint Geršgorin discs, then it concludes that no eigenvalue of $A$ has geometric multiplicity strictly larger than $n+1-m$.

The next theorem is a generalization of Theorem 3.9 in [5]

Theorem 2.7. Let $A \in M_{n}$ and let $\|\cdot\|$ be a matrix norm. Suppose that $\hat{A} \in M_{n-1}$ is a principal submatrix of $A$ such that $\|\hat{A}\|<\|A\|$, and suppose that $\lambda$ is an eigenvalue of $A$ such that $|\lambda|=\|A\|$. Then $\lambda$ has algebraic multiplicity 1. 
Proof. Since $|\lambda|=\|A\|$, Problem 5.6.P38 of [2] ensures that the eigenvalue $\lambda$ is semisimple (algebraic multiplicity equals the geometric multiplicity). Thus, it suffices to show that $\lambda$ has geometric multiplicity 1. If $\lambda$ has geometric multiplicity greater than 1, then Theorem 1.4.10 in [2] ensures that it is an eigenvalue of $\hat{A}$. But, then $|\lambda| \leq\|\hat{A}\|<\|A\|$, which is a contradiction.

Suppose that $A \in M_{n}, \hat{A} \in M_{m}$ is a principal submatrix of $A, B \in M_{m-1}$ is a principal submatrix $\hat{A}$, and $\|B\|<\|\hat{A}\|$. Further assume that $\lambda$ is an eigenvalue of $A$ such that $|\lambda|=\|\hat{A}\|$. If the geometric multiplicity of $\lambda$ as an eigenvalue of $A$ is strictly larger than $n-m+1$, then according to Theorem 2.2, $\lambda$ would be an eigenvalue of $\hat{A}$ with geometric multiplicity strictly larger than 1 , which is in contradiction with Theorem 2.7. Thus the geometric multiplicity of $\lambda$ as an eigenvalue of $A$ must be less than or equal to $n-m+1$.

Theorem 2.8. Let $A \in M_{n}$, let $\|\cdot\|$ be a matrix norm, let $\hat{A} \in M_{m}$ be a principal submatrix of $A$, and let $B \in M_{m-1}$ be a principal submatrix of $\hat{A}$ such that $\|B\|<\|\hat{A}\|$. Further assume that $\lambda$ is an eigenvalue of $A$ such that $|\lambda|=\|\hat{A}\|$. Then the geometric multiplicity of $\lambda$ as an eigenvalue of $A$ must be less than or equal to $n-m+1$.

The following theorem is derived from the Theorem 2.2, along with Theorems 1.1 and 1.2:

Theorem 2.9. Let $A \in M_{n}$, and let $\lambda$ be an eigenvalue of $A$ with geometric multiplicity $k$. Then

(a) $\lambda$ is in at least $m+k-n$ Geršgorin discs of every $m \times m$ principal submatrix $\hat{A}$ of $A$ with $n-k<m \leq n$

(b) $\lambda$ is in at least $m+k-n$ Geršgorin discs of $C_{m+k-n}(\hat{A})$ for every $m \times m$ principal submatrix $\hat{A}$ of $A$ with $n-k<m \leq n$

\section{An upper bound on the absolute value of an eigenvalue}

From the Geršgorin theory, it is known that if $\lambda$ is an eigenvalue of the $n \times n$ matrix $A$, then $|\lambda| \leq\|A\|_{\infty}$. We give an extension of this result.

Theorem 3.1. Let $A \in M_{n}$. For each integer $k, 1 \leq k \leq n$, construct the $n \times n$ matrix $C_{k}$ in the following way: in every row of $A$, replace the smallest 
$k-1$ off-diagonal entries in absolute value by zeros. Let $\alpha_{1}\left(C_{k}\right) \geq \alpha_{2}\left(C_{k}\right) \geq$ $\cdots \geq \alpha_{n}\left(C_{k}\right)$ be the absolute row sums of $C_{k}$ in non-increasing order. Let $\lambda$ be an eigenvalue of $A$. If the geometric multiplicity of $\lambda$ is $k$, then

$$
|\lambda| \leq \alpha_{k}\left(C_{k}\right)
$$

Also, $\alpha_{k}\left(C_{k}\right), k=1,2, \ldots$ is a non-increasing sequence.

Proof. If $\lambda$ has geometric multiplicity $k$, then according to Theorem 1.2 , there are at least $k$ Geršgorin discs of $C_{k}(A)$, say $D_{i_{1}}\left(C_{k}\right), D_{i_{2}}\left(C_{k}\right), \ldots, D_{i_{k}}\left(C_{k}\right)$ with respective centers $c_{i_{1}, i_{1}}, c_{i_{2}, i_{2}}, \ldots, c_{i_{k}, i_{k}}$, and radii $R_{i_{1}}^{\prime}, R_{i_{2}}^{\prime}, \ldots, R_{i_{k}}^{\prime}$ such that

$$
\left|\lambda-c_{i_{j}, i_{j}}\right| \leq R_{i_{j}}^{\prime}, \quad j=1,2, \ldots k .
$$

So,

$$
|\lambda| \leq R_{i_{j}}^{\prime}+\left|c_{i_{j}, i_{j}}\right|, \quad j=1,2, \ldots k .
$$

Hence,

$$
|\lambda| \leq \alpha_{k}\left(C_{k}\right)
$$

Generally, for each $i, 1 \leq i \leq n$, if $1 \leq p \leq q \leq n$, then the $i^{\text {th }}$ disk of $C_{p}$ is not strictly smaller than the $i^{\text {th }}$ disk of $C_{q}$, so

$$
q>p \Longrightarrow \alpha_{q}\left(C_{q}\right) \leq \alpha_{p}\left(C_{q}\right) \leq \alpha_{p}\left(C_{p}\right),
$$

so that $\alpha_{q}\left(C_{q}\right) \leq \alpha_{p}\left(C_{p}\right)$.

Remark 3.2. In each row of $A$, several entries may be equal, so several choices for $C_{k}$ may be possible. Nevertheless, the absolute row sum $\alpha_{i}\left(C_{k}\right)$ is the same for all those choices.

The following corollary follows from the fact that $A$ and its transpose have the same eigenvalues with the same multiplicities.

Corollary 3.3. Let $A \in M_{n}$, and let $\lambda$ be an eigenvalue of $A$ with geometric multiplicity $k$. Let $C_{k}$ and $\alpha_{i}\left(C_{k}\right)$ be defined as in Theorem 3.1, and let $\beta_{i}\left(C_{k}\right)$ be the absolute column-sum of the $i^{\text {th }}$ column of $C_{k}$, for $1 \leq i \leq n$. Then

$$
|\lambda| \leq \min \left(\alpha_{k}\left(C_{k}\right), \beta_{k}\left(C_{k}\right)\right)
$$

For some matrices the upper bound in the formula (1) can be an explicit function of $k$. 
Application 3.4. (Corollary 3.2 in [5]) Let $A \in M_{n}$ be such that

$$
\begin{gathered}
\left|a_{i i}\right|=\beta, \quad i=1,2, \ldots, n \\
\left|a_{i j}\right|=\alpha, \quad i \neq j .
\end{gathered}
$$

If $\lambda$ is an eigenvalue of $A$ with geometric multiplicity $k$, then

$$
|\lambda| \leq \alpha_{k}\left(C_{k}\right)=\beta+(n-k) \alpha .
$$

Example 3.5. $n=5, \beta=0, \alpha=1$ in Application 3.4.

Here, $k=5$ is impossible, since this requires that all off-diagonal entries must be zero, which is not the case. Now,

$$
k=1 \quad \Longrightarrow \quad|\lambda| \leq 4
$$

This is consistent with the fact that $|\lambda|$ cannot exceed any matrix norm of $A$. If $A$ has an eigenvalue $\lambda$ with geometric multiplicity $k \geq 2$, then

$$
k=2 \quad \Longrightarrow \quad|\lambda| \leq 3 .
$$

Example 3.6. Consider

$$
A=\left[\begin{array}{lllll}
0 & 1 & 1 & 1 & 1 \\
1 & 0 & 1 & 1 & 1 \\
1 & 1 & 0 & 1 & 1 \\
1 & 1 & 1 & 0 & 1 \\
1 & 1 & 1 & 1 & 0
\end{array}\right]
$$

Now,

$$
\begin{gathered}
\lambda_{1}=4, \quad k_{1}=1, \quad\left|\lambda_{1}=4\right| \leq \alpha_{1}\left(C_{1}\right)=4 \\
\lambda_{2}=-1, \quad k_{2}=4, \quad\left|\lambda_{2}=-1\right| \leq \alpha_{4}\left(C_{4}\right)=1 .
\end{gathered}
$$

Application 3.7. Let $\left\{P_{i, 1}, P_{i, 2}, \ldots, P_{i, n-1}\right\}=\{1,2, \ldots, n-1\}$ for each $i=$ $1,2, \ldots, n$. Let $A \in M_{n}$ be such that

$$
\begin{gathered}
\left|a_{i j}\right|=r P_{i j} \text { for } i \neq j, \text { for some positive number } r \\
\left\{a_{11}, a_{22}, \ldots, a_{n n}\right\}=\left\{x_{1}, x_{2}, \ldots, x_{n}\right\}, \text { with }\left|x_{1}\right| \geq\left|x_{2}\right| \geq \ldots \geq\left|x_{n}\right| .
\end{gathered}
$$

If $\lambda$ is an eigenvalue of $A$ with geometric multiplicity $k$, then

$$
|\lambda| \leq \alpha_{k}\left(C_{k}\right)=\left|x_{k}\right|+\left(\frac{n(n-1)-k(k-1)}{2}\right) r .
$$


Example 3.8.

$$
\begin{gathered}
A=C_{1}(A)=\left[\begin{array}{rrrr}
1 & 2 & 3 & -1 \\
2 & 1 & -3 & 1 \\
-1 & -2 & 0 & 3 \\
-1 & -2 & -3 & 0
\end{array}\right], \quad C_{2}(A)=\left[\begin{array}{rrrr}
1 & 2 & 3 & 0 \\
2 & 1 & -3 & 0 \\
0 & -2 & 0 & 3 \\
0 & -2 & -3 & 0
\end{array}\right] \\
k=1 \Longrightarrow|\lambda| \leq 7 \\
k=2 \Longrightarrow|\lambda| \leq 6
\end{gathered}
$$

In fact $|\lambda|=2$ is an eigenvalue of $A$ with geometric multiplicity $k=2$

Application 3.9. Let $\left\{P_{i, 1}, P_{i, 2}, \ldots, P_{i, n-1}\right\}=\left\{1,4, \ldots,(n-1)^{2}\right\}$ for each $i=1,2, \ldots, n$. Let $A \in M_{n}$ be such that

$$
\begin{gathered}
\left|a_{i j}\right|=r P_{i j}^{2} \text { for } i \neq j, \text { for some positive number } r \\
\left\{a_{11}, a_{22}, \ldots, a_{n n}\right\}=\left\{x_{1}, x_{2}, \ldots, x_{n}\right\}, \text { with }\left|x_{1}\right| \geq\left|x_{2}\right| \geq \ldots \geq\left|x_{n}\right| .
\end{gathered}
$$

If $\lambda$ is an eigenvalue of $A$ with geometric multiplicity $k$, then

$$
|\lambda| \leq \alpha_{k}\left(C_{k}\right)=\left|x_{k}\right|+\left(\frac{n(n-1)(2 n-1)-k(k-1)(2 k-1)}{6}\right) r
$$

\section{Non-real eigenvalues of real matrices}

In [4], we proved the following.

Theorem 4.1. Let $A$ be a real matrix of order $n$ and let $q$ be a positive integer with $q \geq 1$. If no more than $q$ Geršgorin discs of $A$ form a connected region, then no non-real number can be an eigenvalue of $A$ with geometric multiplicity larger than $\frac{q}{2}$.

An alternative to this theorem is:

Theorem 4.2. Let $A$ be a real matrix of order $n$, let $k$ be a positive integer such that $1 \leq k \leq n$, and let $\lambda$ be a non-real complex number. If $\lambda$ is an eigenvalue of $A$ with geometric multiplicity $k$ or more, then at least $k+1$ Geršgorin discs of $C_{k}(A)$ form a connected region, and $\lambda$ is in the intersection of at least $k$ of them. 
Proof. Choose any principal submatrix $B_{1}$ of $A$ of order $n-k+1$. By Theorem $2.1, \lambda$ is an eigenvalue of $B_{1}$, and hence in one of its G-discs. In the corresponding row (say row $r$ ) of the matrix $A$, we can replace the smallest $k-1$ off-diagonal entries in absolute value by zeros, so that $\lambda$ is in the associated G-disc of the new matrix. Next, delete row and column $r$ from $A$ to obtain a principal submatrix $A_{2}$ of $A$ (which can be considered as $A_{1}$ ) of order $n-1$. Choose any principal submatrix $B_{2}$ of $A_{2}$ of order $n-k+1$. Continue this process until we reach a principal submatrix $A_{k}$ of order $n-(k-1)=n-k+1$, and repeat the procedure on $A_{k}$. This completes $k$ steps in which we have replaced in $k$ rows of $A$ the smallest $k-1$ off-diagonal entries in absolute value by zeros, and $\lambda$ is in each of the corresponding G-discs.

But, since $A_{k}$ is a real matrix with a non-real eigenvalue $\lambda$, the conjugate of $\lambda$ is also an eigenvalue of $A_{k}$, and so $\lambda$ must be in a connected region formed by at least two Geršgorin discs of $A_{k}$. This provides another row of $A$ in addition to the previous $k$ rows, in which we can also replace the smallest $k-1$ offdiagonal entries in absolute value by zeros. Hence, $\lambda$ is in the connected region formed by the corresponding $k+1$ discs. Finally, in each of the remaining $n-k-1$ rows, also replace the smallest $k-1$ off-diagonal entries in absolute value by zeros.

Corollary 4.3. Let $A$ be a real matrix of order $n$, and let $k$ be an integer with $k \geq 1$. If no more than $k$ Geršgorin discs of $C_{k}$ form a connected region, then no non-real number can be an eigenvalue of $A$ with geometric multiplicity $k$ or larger.

For a real matrix $A$ whose G-discs and those of $C_{k}(A)$ have similar sizes, Theorem 4.1 can be conclusive whereas Theorem 4.2 may not be. This may happen when $A$ is sparse.

\section{Example 4.4.}

$$
\text { Let } A=\left[\begin{array}{rrrrr}
1 & \sqrt{2} & 0 & 0 & 0 \\
-\sqrt{2} & -1 & 0 & 0 & 0 \\
0 & 0 & 1 & 0 & 0 \\
0 & 0 & 0 & b_{11} & b_{12} \\
0 & 0 & 0 & b_{21} & b_{22}
\end{array}\right], \quad \text { and let } B=\left[\begin{array}{ll}
b_{11} & b_{12} \\
b_{21} & b_{22}
\end{array}\right] \text {. }
$$

For the matrix $A=C_{2}$, the complex number $i$ is an eigenvalue and the first 3 rows are associated with 3 connected discs. Hence, Theorem 4.2 does not allow us to conclude that the geometric multiplicity of $i$ does not exceed 1 . However, assuming that the entries of the matrix $B$ are such that the two remaining discs of $A$ are disconnected from the 3 previous ones, Theorem 4.1 is conclusive about the fact that the geometric multiplicity of $i$ does not exceed 1. 
Also, if we replace all or some of the zeros in $A$ by real numbers small enough in absolute values for the discs associated with the last 2 rows of A still to be disconnected from the others, continuity of the eigenvalues suggests that one of the eigenvalues of $A$ may be a non-real number equal to or close to the number $i$. In this case, Theorem 4.1 is still conclusive about the fact that the geometric multiplicity of any non-real eigenvalue of $A$ does not exceed 1 , but not Theorem 4.2 since the first 3 discs are still connected in both $A$ and $C_{2}$.

For dense matrices, the difference in size between the discs of $A$ and $C_{k}(A)$ for some integer $k$ can be large enough for the Theorem 4.2 to be conclusive whereas Theorem 4.1 may not be.

Example 4.5. Consider

$$
A=\left[\begin{array}{rrrr}
4.7 & 1 & 0.21 & 0.59 \\
0 & 1.5 & 0 & 1.25 \\
1 & 0.4 & 4.3 & 0.4 \\
0 & -1 & 0 & 0.5
\end{array}\right]
$$

The eigenvalues of $A$ are: $\lambda_{1}=1+i, \quad \lambda_{2}=1-i \quad \lambda_{3}=4, \quad \lambda_{4}=5$.

By looking just at the Geršgorin region of $A$, Theorem 4.1 does not allow us to conclude that $A$ has no non-real eigenvalue with geometric multiplicity larger than 1. But indeed we can deduce the latter fact by invoking Theorem 4.2 and looking at the Geršgorin region of $C_{2}$; see Figures 1,2.

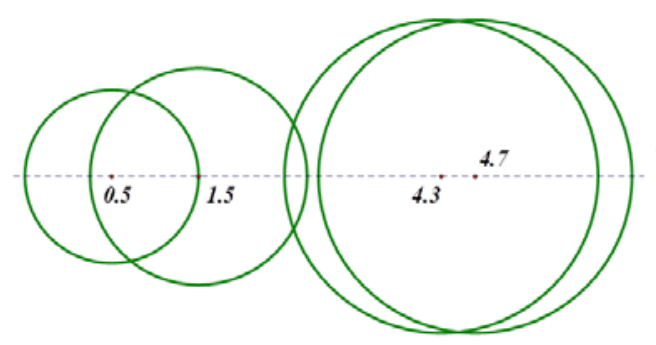

Figure 1: Geršgorin area of $\mathrm{A}$

\section{Connections with powers of matrices}

The next theorem was proved in [4]. 


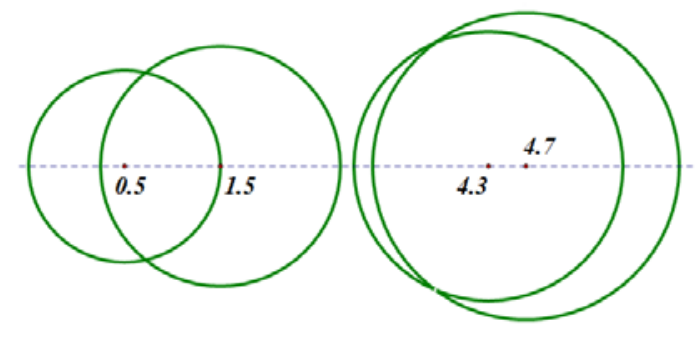

Figure 2: Geršgorin area of $C_{2}(A)$

Theorem 5.1. Let $A \in M_{n}$ and let $\beta, \lambda_{1}, \ldots, \lambda_{s}$ be complex numbers. Suppose that $\beta \neq 0, \lambda_{1}, \ldots, \lambda_{s}$ are distinct eigenvalues of $A$, and $\lambda_{1}^{m}=\cdots=\lambda_{s}^{m}=\beta$. Then, $\beta$ is an eigenvalue of $A^{m}$ and $\beta$ is in at least

$$
\sum_{j=1}^{s} \text { geom mult }\left(\lambda_{j}\right)
$$

Geršgorin discs of $A^{m}$.

We obtain a similar theorem using the matrix $C_{h}\left(A^{m}\right)$.

Theorem 5.2. Let $A \in M_{n}$ and let $\beta, \lambda_{1}, \ldots, \lambda_{s}$ be complex numbers. Suppose that $\beta \neq 0, \lambda_{1}, \ldots, \lambda_{s}$ are distinct eigenvalues of $A$, and $\lambda_{1}^{m}=\cdots=\lambda_{s}^{m}=\beta$. Then, $\beta$ is an eigenvalue of $A^{m}$ and $\beta$ is in at least

$$
h=\sum_{j=1}^{s} \text { geom mult }\left(\lambda_{j}\right)
$$

Geršgorin discs of $C_{h}\left(A^{m}\right)$.

Proof. As in the proof of Theorem 5.1, the geometric multiplicity of $\beta$ as an eigenvalue of $A^{m}$ is $h$. Hence, the result follows by Theorem 1.2.

Example 5.3. Consider the following matrix, which has 1 as an eigenvalue:

$$
A=\left[\begin{array}{rrr}
1 & 1 & 4 \\
1 & -3 & 0 \\
1 & -3 & 5
\end{array}\right]
$$


Then

$$
A^{2}=\left[\begin{array}{rrr}
6 & -14 & 24 \\
-2 & 10 & 4 \\
3 & -5 & 29
\end{array}\right]
$$

Notice that -1 is in some of the $G$-discs of $A$, so it is a potential eigenvalue of $A$. If -1 and 1 are eigenvalues of $A$, then the geometric multiplicity of 1 as an eigenvalue of $A^{2}$ would be at least 2 ; thus, according to Theorem 5.1, 1 would be in at least 2 discs of $A^{2}$ which is not the case. Hence, -1 is not an eigenvalue of $A$.

Example 5.4. Now consider the following matrix A for which 1 is an eigenvalue:

$$
A=\left[\begin{array}{rrr}
1 & 1 & 3 \\
1 & -2 & 0 \\
1 & -2 & 4
\end{array}\right]
$$

Then

$$
A^{2}=\left[\begin{array}{rrr}
5 & -7 & 15 \\
-1 & 5 & 3 \\
3 & -3 & 19
\end{array}\right] \text { and } C_{2}\left(A^{2}\right)=\left[\begin{array}{rrr}
5 & 0 & 15 \\
0 & 5 & 3 \\
0 & -3 & 19
\end{array}\right]
$$

Notice that 1 is in 2 G-discs of $A^{2}$, so that Theorem 5.1 may not help us to decide that -1 is not an eigenvalue of $A$. However 1 is in only one G-disc of $C_{2}\left(A^{2}\right)$; thus, according to Theorem 5.2, - 1 cannot be an eigenvalue of $A$.

\section{Connections with the rank of a matrix}

We first mention a result from [2], 6.1P6, page 394: If $A \in M_{n}$ and $\left|a_{i i}\right|>$ $R_{i}^{\prime}(A)$ for more than $m$ values of $i$, then the rank of $A$ is greater than $m$.

Theorem 6.1. Let $A \in M_{n}$, and let $k$ be an integer with $1 \leq k \leq n$. If $\left|a_{i i}\right|>R_{i}^{\prime}\left(C_{k}\right)$ for more than $n-k$ values of $i$, then the rank of $A$ is strictly greater than $n-k$; equivalently, the geometric multiplicity of 0 as an eigenvalue of $A$ cannot exceed $k-1$.

Proof. If the geometric multiplicity of 0 as an eigenvalue of $A$ exceeds $k-1$, then according to Theorem 1.2, 0 must be in at least $k$ Geršgorin discs of $C_{k}$; this means that 0 is strictly outside of at most $n-k$ discs of $C_{k}$, which is not the case. 
Example 6.2. Consider

$$
A=C_{1}(A)=\left[\begin{array}{rrrr}
3 & 1 & 1 & 1 \\
1 & 3 & -1 & -1 \\
2 & -1 & 4 & -1 \\
1 & 1 & -2 & 2
\end{array}\right], \quad C_{2}(A)=\left[\begin{array}{rrrr}
3 & 1 & 1 & 0 \\
1 & 3 & -1 & 0 \\
2 & -1 & 4 & 0 \\
0 & 1 & -2 & 2
\end{array}\right]
$$

The first column in $A$ is a sum of the remaining columns, which indicates that the number 0 is an eigenvalue of $A$. However, in $C_{2}(A)$, the first 3 diagonal entries are strictly larger than the absolute sum of the off-diagonal entries in the corresponding rows, which allows us to conclude, according to Theorem 6.1, that 0 cannot have geometric multiplicity larger than 1 . Hence, the rank of $A$ is equal to 3 .

\section{Acknowledgement}

The authors wish to express sincere appreciation to Professor Roger Horn for his very useful comments, which greatly improved the manuscript.

\section{References}

[1] M. Fiedler, F. J. Hall, and R. Marsli, Geršgorin Discs Revisited, Linear Algebra and Its Applications 438(2013), 598-603.

[2] R. A. Horn and C. R. Johnson, Matrix Analysis, 2nd ed, Cambridge University Press, Cambridge, 2013.

[3] R. Marsli and F. J. Hall, Geometric Multiplicities and Geršgorin Discs, The American Mathematical Monthly 120(2013), 452-455.

[4] R. Marsli and F. J. Hall, Further Results on Geršgorin Discs, Linear Algebra and Its Applications 439(2013), 189-195.

[5] R. Marsli and F. J. Hall, Some Refinements of Geršgorin Discs, the International Journal of Algebra, 7(2013), 573-580.

[6] R. S. Varga, Geršgorin and His Circles, Springer Series in Computational Mathematics, 36, Springer-Verlag, Berlin, 2004.

\section{Received: January 15, 2014}

